\title{
Visible Nulling Coronagraphy Testbed Development for Exoplanet Detection
}

\author{
Richard G. Lyon**a, Mark Clampin ${ }^{\mathrm{a}}$, Robert A. Woodruff ${ }^{\mathrm{b}}$, Gopal Vasudevan ${ }^{\mathrm{c}}$, Patrick Thompson ${ }^{\mathrm{a}}$, \\ Andrew Chen ${ }^{\mathrm{d}}$, Peter Petrone ${ }^{\mathrm{e}}$, Andrew Booth ${ }^{\mathrm{e}}$, Timothy Madison ${ }^{\mathrm{a}}$, Matthew Bolcar ${ }^{\mathrm{a}}$, M. Charley \\ Noecker ${ }^{\mathrm{f}}$, Stephen Kendrick ${ }^{\mathrm{f}}$, Gary Melnick ${ }^{\mathrm{g}}$, Volker Tolls ${ }^{\mathrm{g}}$ \\ ${ }^{a}$ NASA/Goddard Space Flight Center, Greenbelt, MD 20771; \\ ${ }^{\mathrm{b}}$ Lockheed-Martin, Denver CO; \\ 'Lockheed-Martin Advanced Technology Center, Palo Alto CA \\ ${ }^{\mathrm{d}}$ Lockheed-Martin, Greenbelt MD; \\ 'Sigma Space, Lanham MD; \\ ${ }^{\mathrm{f}}$ Ball Aerospace and Technology Corp, Boulder CO; \\ ${ }^{\mathrm{g}} \mathrm{SAO} / \mathrm{CfA}$, Cambridge MA
}

\begin{abstract}
Three of the recently completed NASA Astrophysics Strategic Mission Concept (ASMC) studies addressed the feasibility of using a Visible Nulling Coronagraph (VNC) as the prime instrument for exoplanet science. The VNC approach is one of the few approaches that works with filled, segmented and sparse or diluted aperture telescope systems and thus spans the space of potential ASMC exoplanet missions. NASA/Goddard Space Flight Center (GSFC) has a well-established effort to develop VNC technologies and has developed an incremental sequence of VNC testbeds to advance the this approach and the technologies associated with it. Herein we report on the continued development of the vacuum Visible Nulling Coronagraph testbed (VNT). The VNT is an ultra-stable vibration isolated testbed that operates under high bandwidth closed-loop control within a vacuum chamber. It will be used to achieve an incremental sequence of three visible light nulling milestones of sequentially higher contrasts of $10^{8}, 10^{9}$ and $10^{10}$ at an inner working angle of $2 * \lambda / \mathrm{D}$ and ultimately culminate in spectrally broadband $(>20 \%)$ high contrast imaging. Each of the milestones, one per year, is traceable to one or more of the ASMC studies. The VNT uses a modified Mach-Zehnder nulling interferometer, modified with a modified "W" configuration to accommodate a hex-packed MEMS based deformable mirror, a coherent fiber bundle and achromatic phase shifters. Discussed will be the optical configuration laboratory results, critical technologies and the null sensing and control approach.
\end{abstract}

Keywords: Exosolar planets, visible nulling coronagraph, visible nulling interferometer, coronagraph, wavefront control, null control, interferometry

\section{INTRODUCTION}

The Extrasolar Planetary Imaging Coronagraph ${ }^{1,2,3}$ (EPIC) (Clampin et. al, 2004, 2006, 2009), Diluted Aperture Visible Nulling Coronagraphic Imager ${ }^{4}$ (DAViNCI) (Shao et. al, 2009 and, the Advanced Technology for Large Aperture Space Telescope ${ }^{5}$ (ATLAST) (Postman et. al, 2010), were three separate NASA Astrophysics Strategic Mission Concept studies (figure-1), each of which assessed the feasibility of using a visible nulling coronagraph ${ }^{6,7}$ (VNC) as their prime instrument for exoplanet detection and characterization. The three studies spanned the space of possible aperture configurations of filled, segmented, and dilute/sparse aperture configurations for which the VNC is well suited. EPIC is a filled aperture 1.65-meter unobscured telescope (figure-1 left). ATLAST (figure-1 middle) assessed several telescope options including a 9.2-metered segmented aperture, an 8-meter filled aperture and 16-meter segmented aperture, while DAViNCl (figure-1 right) is a dilute or sparse aperture telescope with a baseline telescope spacing of 2-meters, The VNC was originally designed and developed for EPIC, but now designs have been put forth for both ATLAST and DAViNCl .

*Richard.G.Lyon@nasa.gov; phone 1301 286-4302 


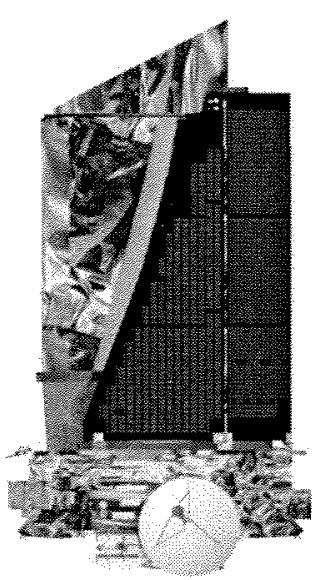

EPIC

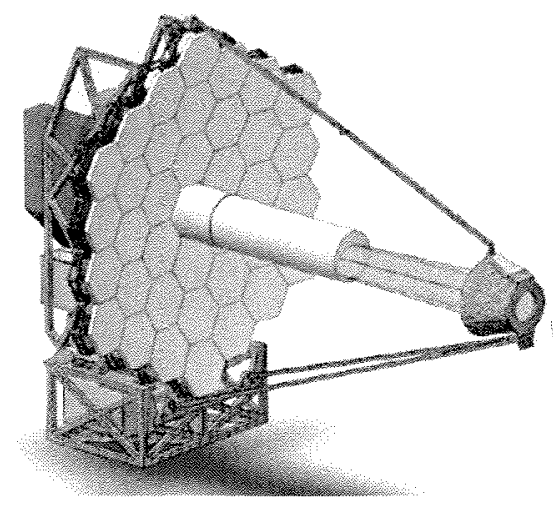

ATLAST

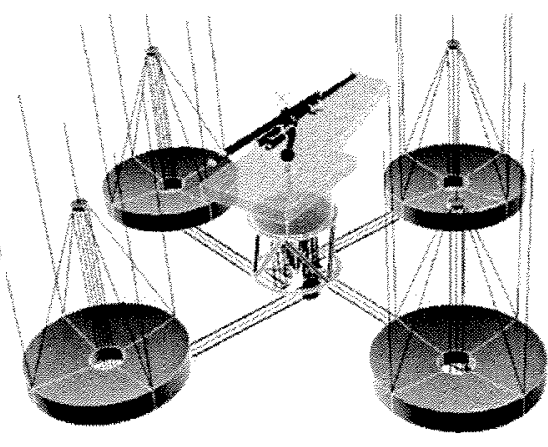

DAVINCI

Figure 1 - Astrophysics Strategic Mission Concepts where a Visible Nulling Coronagraph would enable Exovlanet Science.

EPIC would provide insights into the physical nature of a variety of planets in other solar systems complimenting radial velocity (RV) and astrometric planet searches. It would detect and characterize the atmospheres of planets identified by radial velocity surveys, determine orbital inclinations and masses, characterize the atmospheres around $\mathrm{A}$ and $\mathrm{F}$ stars, observed the inner spatial structure and colors of inner Spitzer selected debris disks. EPIC would be launched to heliocentric Earth trailing drift-away orbit, with a 5-year mission lifetime. ATLAST and DAVINCI would enable direct detection and characterization of exosolar terrestrial mass planets and characterization of their atmospheres and dust/debris disks.

Figure-2 shows one possible design of a VNC instrument followed by a science imaging camera and dispersive spectrometer. In this design the imager and spectrometer are considered as part of the VNC instrument, however, other design

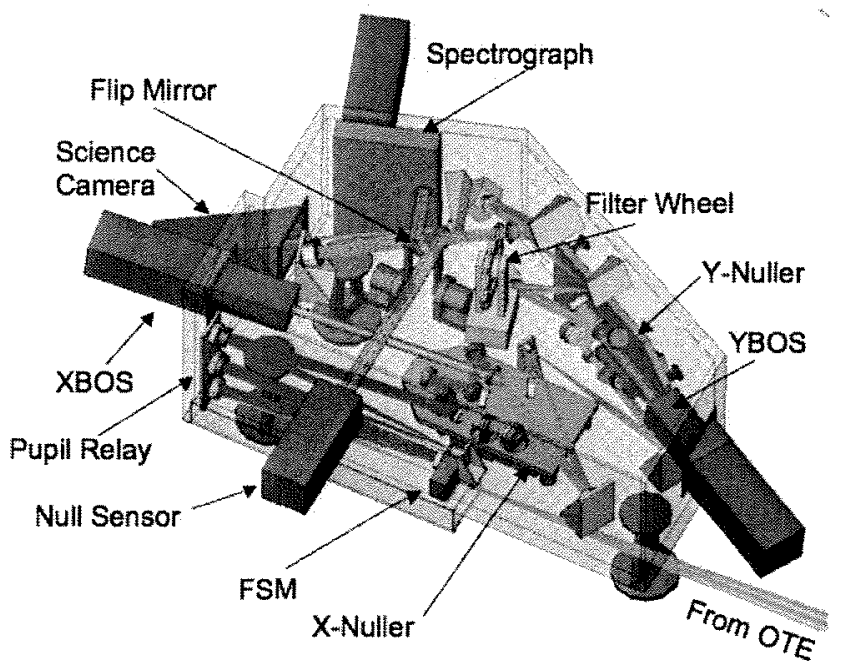

Figure 2 - Visible Nulling Coronagraph Instrument Design configurations are also possible which treat the imager and spectrometer as separate instruments. The critical subsystems of the VNC are the two nullers within it (figure-3). These are shown as the orange bench towards the center of figure-2, and on the right wall of the VNC enclosure in figure-2, and are known respectively as the X- and Y-nullers. These nullers are actually modified Mach-Zehnder interferometers and are the only place within the optical train where the light is interferometrically split, via beamsplitters, into two separate optical paths and subsequently recombined at the output. The nullers effectively perform suppression of the starlight and most of the critical VNC technologies are associated with these nullers. The principle of the $\mathrm{VNC}$ has been adequately described elsewhere and the mathematical model of how it operates is well understood see for example, Lyon et. al, $2006^{6}, 2008^{7}$ and the references therein.

To lower risk, advance technological readiness, quantify performance, develop and assess wavefront and null control algorithms, and assess internal VNC technologies which include MEMS segmented deformable mirrors, achromatic phase shifters, and spatial filter arrays, GSFC has developed a vacuum nulling coronagraph testbed (VNT) based on the design of the nullers shown in figure-3. Past work has concentrated on proof of principle and we have subsequently demonstrated nulling ${ }^{9}$ and the next phase of the VNT efforts are concentrated on quantification of its performance and 
advancing and refining its control approach to push towards broadband visible nulling approaching the $10^{10}$ contrast level. Recent the VNT was moved to the new state-of-art lab facilities at/GSFC is undergoing a major retrofit.

Herein we first describe the retrofitted VNT and the milestones to be achieved in 2010 and 2011 and the tracebility of the milestones to the ASMC missions.

\section{VNT DESCRIPTION}

\subsection{Description of the Visible Nulling Testbed}

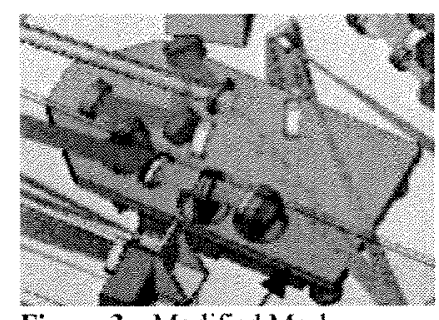

Figure 3-Modified MachZehnder Nuller.

A schematic drawing of the VNT testbed is shown in figure-4 and described here. The VNT resides on a 24 " $\mathrm{x} 36$ "

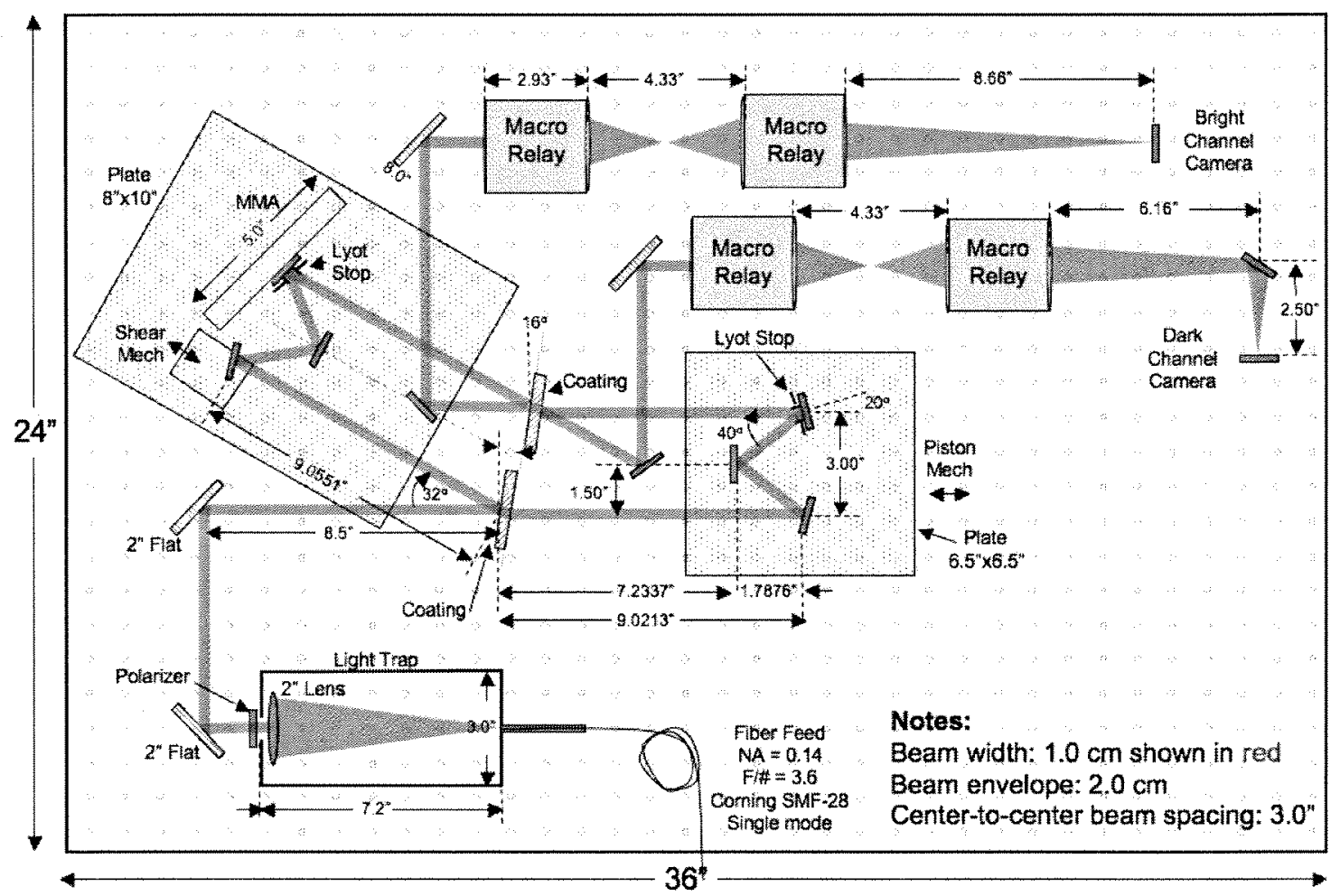

Figure 4-Schematic of Visible Nulling Testbed (VNT).

optical table. Light enters from a supercontinuum source outside the vacuum chamber through a single mode fiber at the bottom center of figure-4. Light exits the single mode optical fiber as approximately an expanding Gaussian beam into a tubular light trap with black absorbing material. Light can only exit at the left face of the light trap through a 2 " apochromatic collimating lens. The lens is stopped down to let light only over the central $2 \mathrm{~cm}$ aperture diameter exit. This effectively allows only $\sim 1 / 3$ of the central diameter of the Gaussian beam to exit insuring a nearly perfect wavefront and amplitude quality, i.e. a high order approximation to a plane wave. A linear polarizer is within the exit port of the light trap with the polarization axis set such that the E-field vector is pointing out of the plane of figure- 4 . This is to insure initially that all reflective optics sees transverse electric (TE) mode fields to minimize polarization cross-leakage. The polarizer is removable so that we can quantify the difference in contrast between polarized and unpolarized light. The symmetry of the nuller makes it in principle insensitive to the polarization state of the input field, however differences in coatings will introduce some asymmetry. 
After the light trap are two alignment flats that are used to steer the beam onto the first beampslitter at an angle of incidence of 32 degrees. There are two custom matched beamsplitters made of high-grade fused silica with dielectric coatings, the first is used to split the beam and the $2^{\text {nd }}$ to recombine. The $1^{\text {st }}$ beamsplitter has its coated side facing the incident light while the $2 n$ beamsplitter is flipped with the coating side facing in the opposite direction. At the $1^{\text {st }}$ beamsplitter the light is split into a reflective path towards the upper left and a transmissive path towards the right. The transmissive path reflects off three flat optics that are mounted in a "W" configuration. The "W" is aligned such that the input and output beams are parallel and is built and aligned as a separate rigid assembly. This assembly is

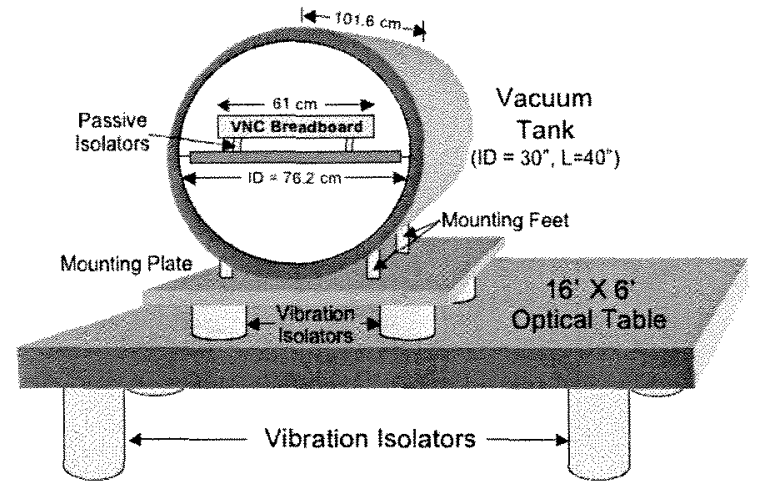

Figure 5 - VNT Lab Configuration mounted on a two stage (coarse and fine), 1 degree of freedom (DOF) translation stage known as the piston mechanism. This mechanism is used to change the overall path length in the transmissive arm reflective to the reflective arm of the nuller, this path length difference is controlled actively during closed-loop operations.

\subsection{Test Configuration}

Figure-5 shows a simple schematic of the testbed configuration resting on the isolation table. The feet of the table are $\sim 1 \mathrm{~Hz}$ air based isolators. The vacuum tank rests on passive isolators on the top of the table and the VNT rests on a shelf on top of passive isolators in the vacuum tank. There is an optical feed through on the right wall of the tank and all the electrical feedthroughs are on the left wall of the tank. A water based chiller system cools the camera. Figure- 6 shows the previous version of the VNT resting on a table (left) and within the vacuum tank (right).

\subsection{Description of the Multiple Mirror Array}

The reflective arm of the nuller also traverses a "W" configuration towards the upper left, however the first optic is mounted on a shear mechanism. The shear mechanism is a 1-DOF translation stage that translates along the incident beam direction. The effect of this motion, after passing through the "W", is to walk or shear the beam sideways relative to the beam in the nullers other arm. The $3^{\text {rd }}$ optic in this nuller is a micro-electrical-mechanical (MEMS) deformable mirror with a segmented facesheet known as a multiple mirror array (MMA) as shown in figure-7. Each of the MMA segments are individually actuated with biomorph flexure actuators such that each of the segments moves as a rigid body in piston, tip and tilt (3-DOF). The MMAs function is to minimize the wavefront difference between the two arms of the
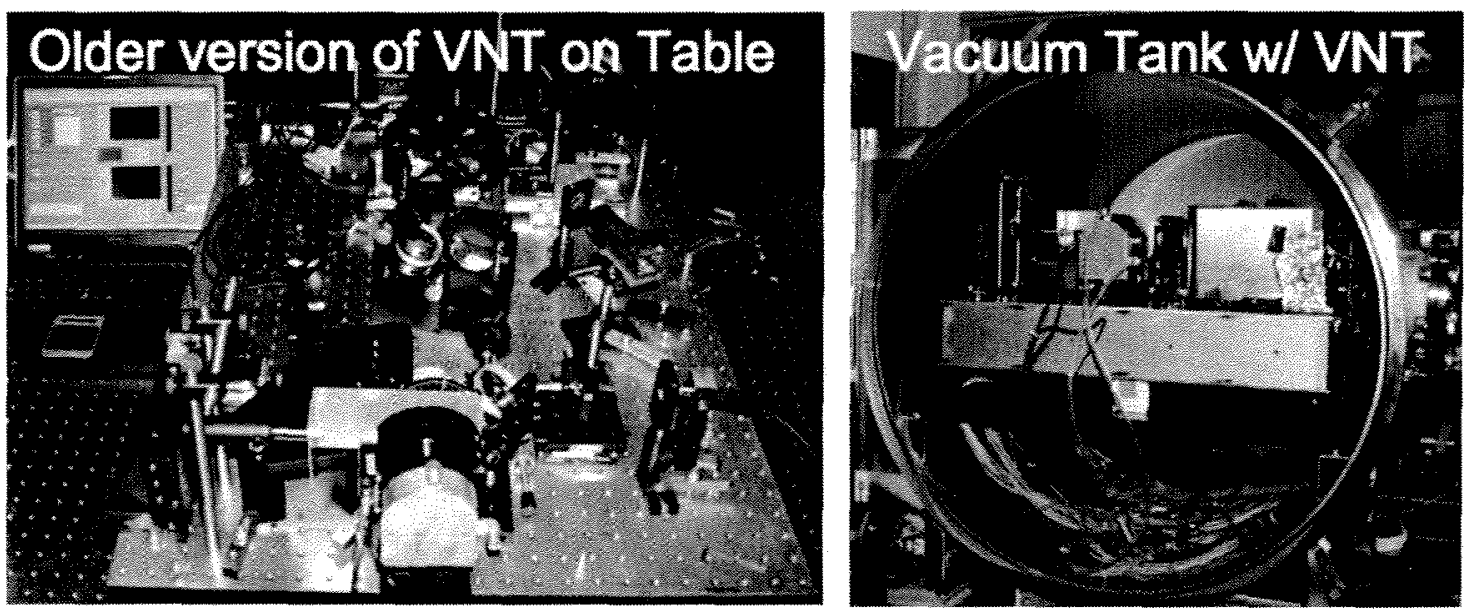

Figure 6-Left: Older version of VNT resting on table, right: Older version of VNT in Tank. 
nuller. The MMA operates in closed-loop in conjunction with the piston mechanism, where the mean motion of the aggregate set of MMA segments is integrated and offloaded to the piston mechanism at lower bandwidth of $\sim 1 \mathrm{~Hz}$. This is to keep the MMA from using up its stroke range in compensating for overall piston differences in the two arms of the nuller.

Both the transmissive and reflective arms of the nuller are combined at the $2^{\text {nd }}$ beamsplitter and yields two output beams known as the bright and dark channels as labeled in figure-4. Only the dark channel is truly symmetric since it sees a coherent sum of a beam that 1 st reflects off beam splitter 1 and subsequent transmits through beam splitter 2 coherently added to the beam which transmits through beam splitter 1 and reflects of beam splitter 2 , i.e. $E_{D} \propto r t+r t e^{i \pi}=0$ without any wavefront error and where the $\pi$ phase shift is introduce by the path length difference between the two nuller arms. The bright channel sees a coherent sum of a beam that reflects off both beamsplitters added to a beam that transmits through both beamsplitters, i.e. $E_{B} \propto r^{2}+t^{2}$ and is thus more sensitive to imbalance in the reflection and transmission of the two beamsplitters. The bright and dark channels pass through two macro lens groups which for the bright channels relays an image of MMA onto a 16-bit CMOS high frame rate detector, i.e. a pupil image. The dark channel detector sees a focal plane image on a photon counting cooled E2V camera. All the optics which appear in the non-common path arms of the nuller are high quality flat optics with fused silica substrate and coated with the same broadband coatings except for the MMA and its counterpart in the other arm. The MMA is coated with protected aluminum and its counterpart flat is also coated with protected aluminum from the same coating vendor and with the same coating prescription to balance reflectivities. Additionally the MMA has segment gaps which are not resident in the other arm of the nuller. The segment gaps introduce Fresnel diffraction effects that are different from the other arm. To correct this problem a set of two matched Lyot stops are manufactured that matches the shape of the MMA segment gaps and one is placed a few $\mathrm{mm}$ 's in front of the MMA and the other a few mm's in front of the MMAs counterpart optic in the other arm. These are labeled as Lyot stop in figure-4. The purpose of the Lyot stops is to balance the Fresnel diffraction effects in the system. The location of the Lyot stop is the defined exit pupil of the system.

\subsection{Description of the Spatial Filter Array}

In-between the two-macro lenses of the dark output channel is an assembly for the spatial filter array (SFA). This is not shown explicitly in figure- 4 but it is mounted on a special mount that allows it to be removed and inserted with minimal realignment. Initially the SFA will not be used to attain the $1^{\text {st }}$

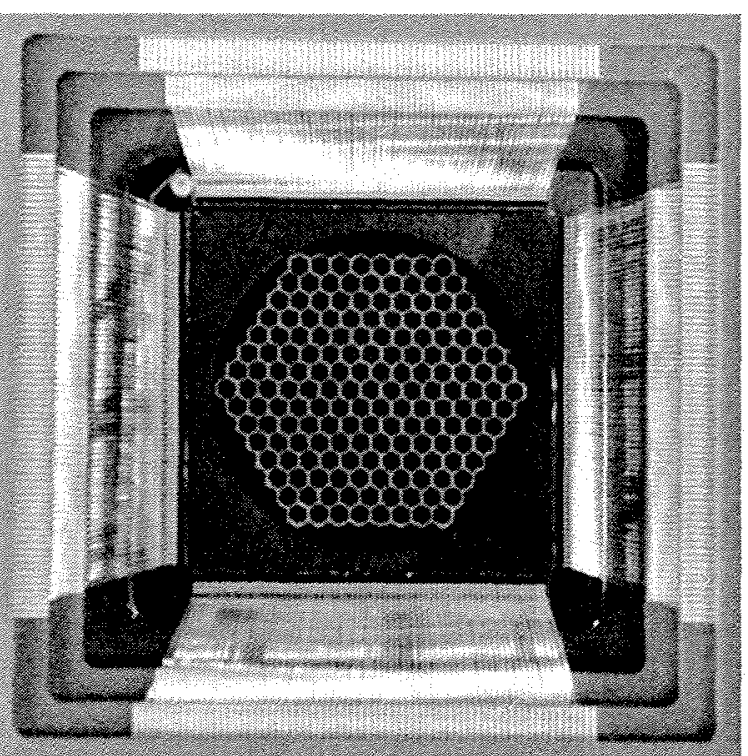

Figure 7 - Photo of Multiple Mirror Array (MMA) 169 segments, 163 are active, outermost 6 inactive. Each is $\sim 700$ microns point-to-point, gaps are $3-5$ microns. Full width of active area is $\sim 8.5 \mathrm{~mm}$. Segment are 50 -um thick single crystal Si overcoated with protected $\mathrm{Al}$, each moves in 3-DOF. Surface is photographed reflecting black cardboard to accent gap locations.

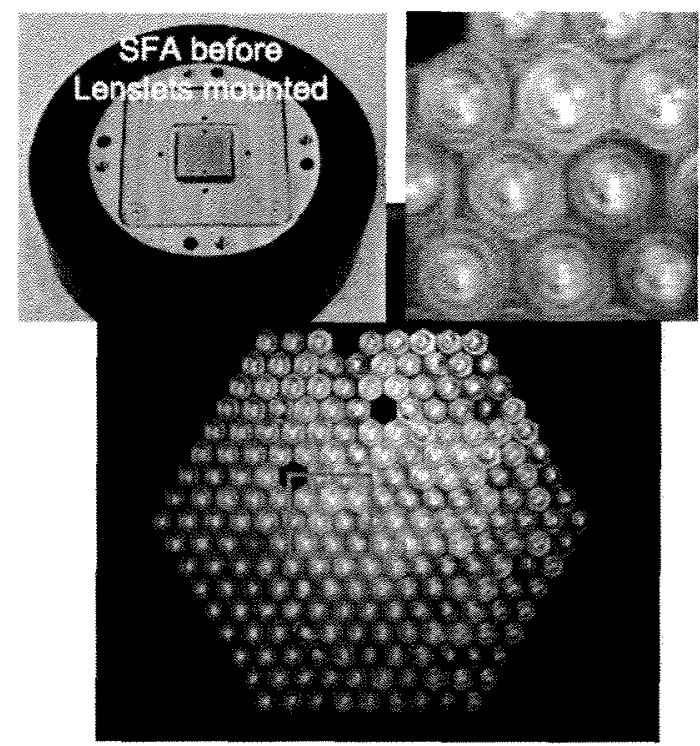

Figure 8 - Spatial Filter Array (SFA). Upper left photo of SFA before lenslet arrays mounted. 217 active fibers in hex-pack. Bottom - Diffuse white light transmitted through SFA, note 3 dead fibers. Upper right - region of inset which shows ring like structure. Each lenset is 500 microns flat-to-flat. 
milestone but it will be tested on a $2^{\text {nd }}$ testbed known as the null control breadboard (NCB) (figure-9). The recently delivered SFA is shown in figure- 8 and consists of an input lenslet array follow by an array of single mode fibers followed by an output array of lenslets. Each input lenslet is mapped to one fiber and to one output lenslets and each lenslet-fiber-lenslet is optically mapped to one MMA segment. When used passively the MMA cleans up the wavefront errors on the scale of 1 cycle per MMA segment and higher since it will be spatially filtered by the fiber. When used in conjunction with the tip/tilt control of the MMA the PSF of a single segment can be steered on the end of the fiber to lower the coupling efficiency into the fiber. Since the MMA is in only one arm of the MMA it only affects that component of the beam and if the other arm is slightly darker then mis-steering can be used to balance the amplitude between the beams. Thus the MMA with SFA can in principle control both amplitude and wavefront errors simultaneously. This principle will be validated and its performance assessed.

\subsection{Null Control Breadboard}

A separate testbed was developed to test the SFA and the MMA and to develop the sensing and control algorithms. It consists of a white-light Michelson interferometer as shown in figure-9. Light enters from a fiber coupled to a supercontinuum light source at the bottom center and passes through a collimating lens to a glass cube beamsplitter which splits into two paths. The path to the right reflects of the MMA and the path to the top off a high quality reference flat. The beamsplitter recombines the beams that pass to the left through the SFA, and one (or more) lenses to ND filters and spectral filters to an imaging CMOS camera that can be framed as fast as 106 frames per second. The mounted MMA can be seen in figure-9. The MMA is mounted on an $X, Y, Z$ translation mount while the reference flat is mounted on a tip/tilt stage. The output images, either in focus or at an image of the

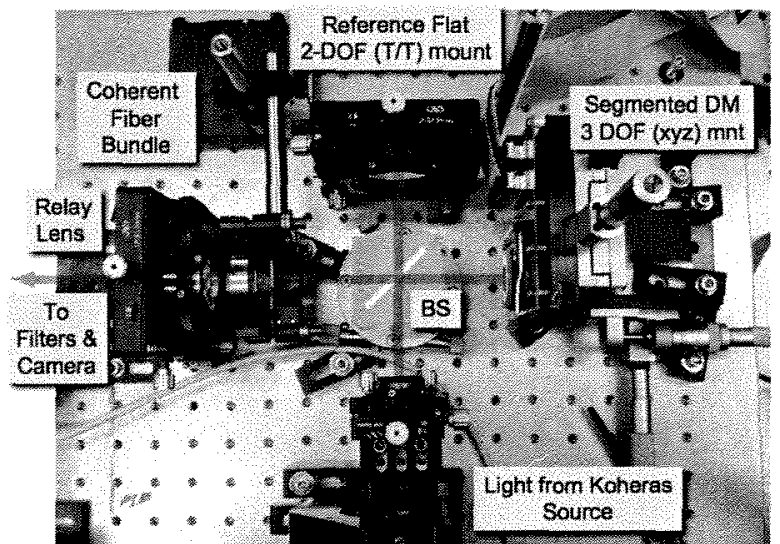

Figure 9 - Null Control Breadboard. MMA can be used to test and control the MMA and the algorithms and algorithms that feedback to the MMA have been tested. The MMA mount is set up to accommodate both IRIS-AO and Boston Micromachines MMAs.

Figure-10 shows a first attempt at nulling one MMA segment against one SFA fiber by dithering the DM mechanism in approximately $1 / 4$ wave increments through 1 wavelength of light. Initially the fiber output is bright then grows dark and subsequently bright again as expected. The next step is quantifying the depth of null with and without the fiber bundle and to measure its throughput, and temporal coherence and spatial coherence transfer functions.

The VNC relies on destructive interference to cancel, or null, on-axis starlight but such that off-axis planet light sees an additional phase shift, introduced by the lateral shear. This effectively increases the contrast of the planet to star at small angular separations and the VNT is designed to assess the performance of this contrast.
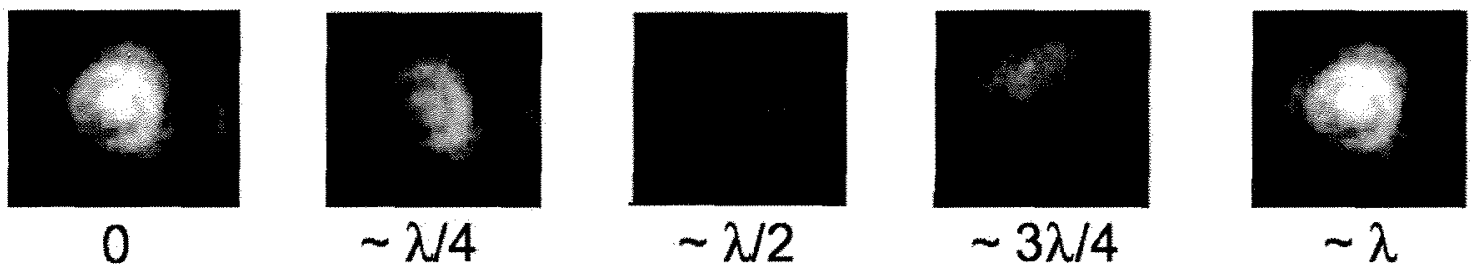

Figure 10 - Example of First Nulling through a Single Fiber of SFA. 


\subsection{Real Time Control System Architecture}

The laboratory environment is likely to be less stable than a spacecraft in L2 or Heliocentric drift-away orbit and two lab development philosophies are possible. The $1^{\text {st }}$ is to attempt to stabilize the environment to match that expected in space, and the $2^{\text {nd }}$ is to control at a bandwidth that is higher than the characteristic frequencies of the lab environment. In reality any lab testbed is likely to trade off these two approaches. For the VNT we operate in a vacuum tank on an isolation table, and control as fast as the technology and cost allow. This necessitated the development of a control architecture with minimal latency and fast processing.

Figure-11 shows, at the top level, the real time control architecture. The heart of it is a control computer with dual hyper threaded hex-core processors that allows two threads of execution per core for 24 threads of execution. This system runs a real time Xenomai Linux kernel within Red-Hat Linux. LabView-8 for Linux runs within this kernel and all the sensing and control functions are

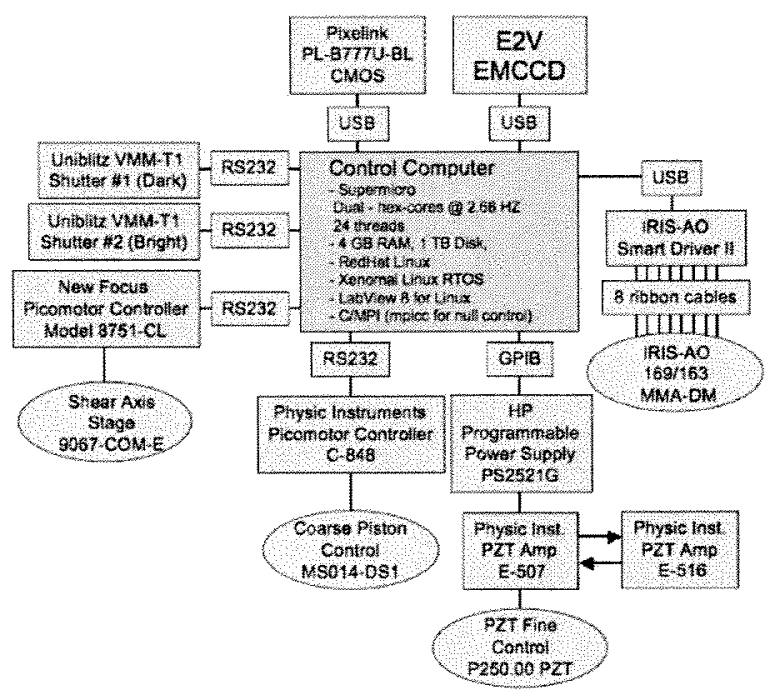

Figure 11 - Real Time Control Architecture written in $\mathrm{C}$ with message passing interface (MPI). MPI is a parallel processing paradigm that allows for one $\mathrm{C}$ main function to execute but have inter thread communications, and data sharing but with different commands executing on each thread. When running in conjunction with the Xenomai is allows timing between the threads to be tightly controlled. The architecture is setup such that a different set of threads operate the cameras, another set of threads perform the wavefront sensing algorithms, and another set of threads control the MMA in a bucket brigade fashion. Thus, while the cameras are collecting data at time $t$, the wavefront sensing is operating on images from time $t-\Delta t$, and the MMA is being commanded based on the images from time t$2 \Delta \mathrm{t}$. This required that a custom device driver be written for the MMA. In practice it is not quite this simple since the CMOS camera (bright channel) and the EMCCD camera (dark channel) will need to operate at different frame rates to balance the sensing and control algorithms. Ultimately we expect the control system to operate in closed-loop at $5-15 \mathrm{~Hz}$.

\subsection{Wavefront Control}

The wavefront control lies at the heart of this entire effort. It is critical to be able to optimally sense and control the wavefront and amplitude errors, both broad and narrowband, at high enough bandwidth to achieve and hold the
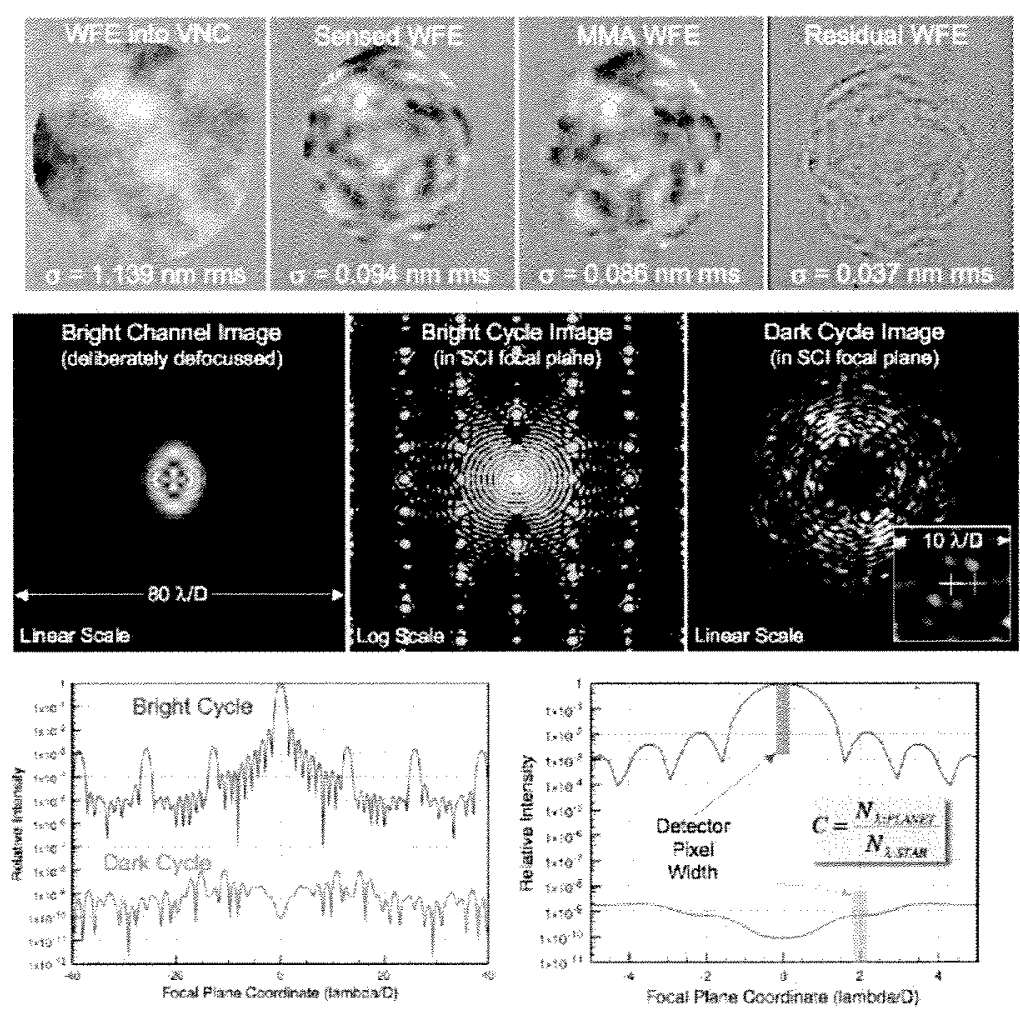

Figure 12 - Wavefront Control Simulations 
contrast. The stroke of the MMA's, bit depth of the cameras, vibration, thermal drift, coating imperfections, temporal and spatial sampling, quantization, flat fielding, dark current, noise, straylight, approximations and errors in the algorithms, along with other potential other potential effects, all work to corrupt this process. The wavefront control is really a multi-step process that consists of a set of camera calibration algorithms, wavefront sensing algorithms and MMA control algorithms. Wavefront sensing though is somewhat of a misnomer since that which is important to the science is the contrast and thus the final control metric needs to be based on this, as opposed to converting images to wavefront. We will not describe herein the mathematical development of the algorithms since this would require at least one entire manuscript unto itself. However we will briefly describe the modeling efforts and show some of the results. The modeling is important since we must ultimately validate the model against the testbed in order to use the model to predict the performance of a flight system - ultimately it is this validated model which is one of the more important aspect of this effort.

Figure-12 shows a simulation of one of the possible wavefront control sequences we will try as part of this effort. The top row left shows an example of a wavefront error at the input to the VNT of $1.139 \mathrm{~nm} \mathrm{rms}$ wavefront error (WFE). This WFE is sheared by a $1 / 4$ pupil, which lowers the $\mathrm{rms}$ since the shear is approximately like a discrete derivative of the wavefront error that lowers the low spatial frequency contribution to the wavefront sigma. The sensed wavefront is shown as the $2^{\text {nd }}$ from the left of the top row and it has a sensed rms of $0.094 \mathrm{~nm}$ after 5 simulated control steps. The $2^{\text {nd }}$ from the right shows the WFE decomposed into the control modes of the MMA at $0.086 \mathrm{~nm}$ rms and the right shows the uncorrected residual of $0.037 \mathrm{~nm} \mathrm{~mm}$ after 5 control steps. This simulation contained the effects of camera sampling, detector readnoise and dark noise in the bright camera channel (left middle row), and photon and dark current noise in the dark camera channel (middle of middle row). After 5 control steps the resulting dark channel image is shown on the right of the middle row. Note that no disturbances were introduced during the 5-step control process during this simulation.

The initial starting WFE is in practice expected to be much higher but a series of coarse control steps will bright it into the range of $<10 \mathrm{~nm}$. This includes an interferometer type algorithm using the bright channel image since this image will be an interferogram of beams in the two arms of the nuller. Also a bright/dark direct DM approach will bring the WFE down to $\sim 1 \mathrm{~nm}$ rms prior to the fine control starting. It is expected that will the current configuration we can achieve and hold $\sim 0.070 \mathrm{~nm}$ rms WFE.

\section{SUMMARY}

The direct detection of exosolar Jovian planets is a crucial step in the search for terrestrial planets with liquid water, and potentially life, and addresses the NASA Search for the Origins of Life theme. Jovian planets, while expected to be further from their parent star and are generally brighter than a terrestrial planet, are still difficult to directly detect. Finding and charactering numerous jovian planets and their parent star's dust disks and comparing them is the work of proposed NASA missions such as the Extrasolar Planetary Imaging Coronagraph (EPIC) and detection and characterization of Earth mass terrestrial planets is the work of proposed missions such as DAViNCI and ATLAST among others. These three missions rely on the advancement of the Visible Nulling Coronagraph and its associated technology and this is being accomplished through the use of past, present, and future laboratory interferometry testbeds. These testbeds are an important step for advancing technology and retiring risk for those technologies (nulling and wavefront control) considered critical to exosolar planet detection.

NASA/Goddard Space Flight Center has developed high stability and vacuum facilities for the visible nulling coronagraph. These facilities were funded under NASA Internal Research and Development over the preceding two years and are now funded under the NASA Technology Development for Exoplanet Missions (TDEM). We expect to achieve repeatable and verifiable contrasts of $10^{8}$ at an inner working angle of $2 \lambda / D$ on the VNT by fall of 2010 and are expected to achieve $10^{9}$ to advance the NASA technology readiness levels for the VNC for exosolar planet detection. Future efforts will concentrate on using higher fidelity deformable mirrors, better-designed achromatic phase shifters, developing of null control algorithms, and increasing the spectral passband and evaluation of spectroscopy through the VNC. 


\section{REFERENCES}

[1] M. Clampin, R. G. Lyon, G. Melnick, P. Nisenson, R. A. Woodruff, M. Harwit, D.Y. Gezari, L. Petro, H. Ford, R. Mauk, H. Smith, "The Extra-solar Planetary Imaging Coronagraph", Proceedings of SPIE 5487, Glasgow Scotland, June $21-25$ (2004).

[2] M. Clampin, G. Melnick, R. Lyon, S. Kenyon, D. Sasselov' V. Tolls, H. Ford, D. Golimowski, L. Petro, G. Hartig, W. Sparks, G. Illingworth, D. Lin, S. Seager, A. Weinberger, M. Harwit, M. Marley, J. Schneider, M. Shao, M. Levine' J. Ge, R. Woodruff, "Extrasolar Planetary Imaging Coronagraph (EPIC)", Proceedings of SPIE 6265, Orlando FL, May (2006).

[3] M. Clampin, R. G. Lyon, G. Melnick, V. Tolls, M. Shao, M. Levine, , R. A. Woodruff, G. Vasudevan, S. Kendrick, "The Extra-solar Planetary Imaging Coronagraph", Mission for Exoplanets Workshop, Pasadena CA, April 21-23, (2009).

[4] Shao, M., Bairstow, S. H., Deems, E., Fletcher, L., Levine, B., Orton, G., Vasisht, G., Wayne, L., Zhao, F., Clampin, M., Lyon, R., Guyon, O., Lane, B., Havey, K., Wynn, J. Samuele, R., Vasudevan, G., Woodruff, R., Tolls, V. Malbet, F., Leger, A., "A Mission Concept Study of a Dilute Aperture Visible Nulling Coronagraph Imager (DAViNCI) for the Detection and Spectroscopy of Exo-planets," BAAS \#214, (2009).

[5] Postman, M., "The Science Cases for an Advanced Technology Large-Aperture Space Telescope (ATLAST)," BAAS \#215, (2010).

[6] R. G. Lyon, M. Clampin, R. Woodruff, G. Vasudevan, M. Shao, M. Levine, G. Melnick, V. Tolls, P. Petrone, P. Dogoda, J. Duval, J. Ge, "Visible Nulling Coronagraphy for Exo-Planetary Detection and Characterization", IAU Colloquim 200, Direct Imaging of Exoplanets: Science and Technology, Villefranche-sur-Mer, France, (2006).

[7] R. G. Lyon, M. Clampin, G. Melnick, V. Tolls, R. Woodruff, G. Vasudevan, "Extrasolar Planetary Imaging Coronagraph (EPIC): Visible Nulling Coronagraph Testbed Results”, Proceedings of SPIE 7010, Marseille Fr, (2008).

[8] R. A. Woodruff, M. Shao, B. Martin Levine, R. Lyon, G. Vasudevan, J. Acu, K. Havey, J. Wynn, "Optical Design of Dilute Aperture Visible Nulling Corongraph Imager," Proc of SPIE 7731, (2010).

[9] R. G. Lyon, M. Clampin, R. Woodruff, G. Vasudevan, P. Thompson, P. Petrone, T. Madison, M. Rizzo, G. Melnick, V. Tolls, "Visible Nulling Coronagraph Testbed Results," Proc of SPIE 7440 (2009). 